\title{
FULLY AUTOMATED REGISTRATION OF 3D CAD MODEL WITH POINT CLOUD FROM CONSTRUCTION SITE
}

\author{
Changmin Kim, Joohyuk Lee, Minwoo Cho, and Changwan Kim* \\ Department of Architectural Engineering, Chung-Ang University, Seoul, Korea \\ *Corresponding author (changwan@cau.ac.kr)
}

\begin{abstract}
In construction automation applications, registration between the 3D computer-aided design (CAD) model and the point cloud obtained by remote sensing technology is an important process such as progress monitoring and as-built modeling. However, the registration method to align the 3D CAD model and point cloud in construction automation has limitations because the registration is performed manually, which is less accurate and more time-consuming. In this paper, an automated registration method for a 3D CAD model with point cloud from the construction site is proposed. Preprocessing is presented to convert points into suitable representation for registration. Then, an automated registration method that determines transformation parameters is presented. Field experiments have been conducted to test the proposed registration method and the results show that the proposed method is useful for construction automation.
\end{abstract}

Keywords: Iterative Closest Point, Principle Component Analysis, 3D Registration

\section{INTRODUCTION}

Registration is a process by which the data acquired from different conditions such as time, viewpoint, sensors and source are transformed and aligned with the same coordinate system [1, 2]. Registration is a fundamental to perform various applications in computer vision such as change detection, object recognition, industrial automation and inspection [3, 4]. In construction automation, registration of the 3D computer-aided design (CAD) model with the point cloud obtained from the construction site is an important process for applications such as progress monitoring and as-built modeling [5, 6, 7]. To perform progress monitoring, the 3D CAD model which contains schedule information, needs to be aligned with the point cloud, which is obtained from structural components, for comparing two data sets. In as-built modeling, the 3D CAD models must be registered with a 3D as-built point cloud to match and retrieve 3D CAD components.

Although registration is useful in construction automation, the current registration of the 3D CAD model with the point cloud method is inefficient. Registration in construction automation is customarily performed manually which can provide inaccurate results due to human error and users' subjective interpretations. The process is also time-consuming because the point cloud acquired from the construction site is large. These problems limit the usage of point cloud that is obtained from the construction site.

To minimize manual intervention during the registration process, [6] proposed a semi-automated registration method for aligning 3D CAD model and point cloud obtained from steel structures. In this research, n-point registration where the user manually selects more than three pairs of corresponding points in the 3D CAD model and point cloud, was used. The iterative closest point algorithm was then used to refine the results of the n-point registration. However, the n-point registration forces the user to select several control points to obtain reliable results, which can be tedious and labor-intensive [8].

In this paper, an automated registration method of a 3D CAD model with point cloud obtained from the construction site is proposed. Proposed registration method explained in detail in the "Research Methodology" section. Using the proposed method, field experiments have been conducted using 3D CAD model and photogrammetric data obtained from the construction site. 


\section{RESEARCH METHODOLOGY}

The registration process of the $3 \mathrm{D}$ CAD model and point cloud is composed of three steps. The first step is preprocessing, which converts points into a suitable representation for 3D registration. Then, the 3D registration estimates the transformation parameters to align model points and data points. The 3D registration can be divided into coarse and fine registration. A detailed description of the methodology is as follows.

\subsection{Preprocssing}

The 3D CAD model and point cloud that are obtained by remote sensing technology is represented by each format. To perform registration, the 3D CAD model and point cloud must be converted into the suitable representation. The 3D CAD model itself is exported in a stereolithography (STL) format consisting of normal and triangular facets. Uniform resolution model points are then generated based on the triangular facets. However, point cloud obtained by remote sensing technology, such as laser scanner or photogrammetry technology, is represented by multiresolution point cloud, because the resolution of point cloud is dependent on the distance between the object and sensor. It is difficult to obtain accurate registration results when two data sets have different resolutions [9]. To remedy this, the model points and point cloud were subjected to a data re-sampling process in order to improve registration accuracy.

In this research, an octree representation was used to make regular points for data re-sampling. An octree is a treestructure that can be used to represent volumetric data surrounded by a bounding cube. The initial cube that is created using the boundary of the object is subdivided into eight sub-cubes (octants), which are then repeatedly subdivided several times, until each sub-cube contains no points, or until the results reaches the criteria defined by the application [10].

The re-sampling process is fourfold. First, each octree of the original data is generated through repeated subdivision as previously described. Second, a list of sub-cubes that contain the points, central points, and the cube lengths is generated. Sub-cubes that are larger than the criteria are deleted from the list because they are unnecessary. Third, all cubes with fewer points than the minimum requirement are removed from the list. This is done to reduce noise, which is an obstacle in robust registration. A cube will also be removed if it contains no points. Finally, the central points of all cubes are extracted, and the point cloud will have a uniform resolution.

\subsection{D Registration}

In this research, registration of the model points with data points consists of coarse and fine registration. In coarse registration, principal component analysis (PCA) is used to estimate the transformation parameter [11]. PCA provides a rough estimate by using only three vectors as principal components. Thus, to obtain the most accurate transformation parameters, the iterative closest point (ICP) was used as fine registration.

\subsubsection{PCA Based Coarse Registration}

PCA uses the direction of the principal axis of point cloud to perform registration [12]. The direction of the principal axis is defined as three eigenvectors of the covariance matrix of the point sets [11]. The covariance matrix $\mathrm{K}$ for given $N$ points $x_{i}=\left(\begin{array}{lll}x_{i} & y_{i} & z_{i}\end{array}\right)^{\mathrm{T}}, \quad i=1, \ldots, N$ is defined as:

$$
\mathrm{K}=\frac{1}{N} \sum_{i=1}^{N}\left\{\left(x_{i}-\bar{x}\right)\left(x_{i}-\bar{x}\right)^{T}\right\}
$$

where the center of points $\bar{x}=\left(\begin{array}{lll}\mu_{x} & \mu_{y} & \mu_{z}\end{array}\right)^{\mathrm{T}}$ $=\frac{1}{N} \sum_{I=1}^{N} x_{i}$.

Let $u_{1}, u_{2}, u_{3}$ and $\lambda_{1}, \lambda_{2}, \lambda_{3}$ be eigenvectors and eigenvalues of $K$, respectively $\lambda_{1} \geq \lambda_{2} \geq \lambda_{3}$. Then, $K$ can be factorized:

$$
\mathrm{K}=U \Lambda U^{\mathrm{T}}
$$


where $U=\left[u_{1}, u_{2}, u_{3}\right]$ and $\Lambda=\operatorname{diag}\left(\lambda_{1}, \lambda_{2}, \lambda_{3}\right)$.

The rotation matrix $R$ can be estimated from:

$$
R=U_{m} U_{d}^{-1}
$$

where $U_{m}$ and $U_{d}$ are eigenvectors of model points and data points.

The translation vector $t$ can be estimated by calculating the distance between the center of the model and the data points.

$$
t=\bar{x}_{m}-R \bar{x}_{d}
$$

where $\bar{x}_{m}$ and $\bar{x}_{d}$ are the center of the model points and data points, respectively.

\subsubsection{LM-ICP Based Fine Registration}

The ICP algorithm proposed by [13] is widely used to perform fine registration in two steps [14]. First, it generates correspondence between two point sets such that each set corresponds with the nearest point in the other point set. Then, it computes the new transformation parameters by minimizing the Euclidean distance between corresponding point pairs. These two steps are iterated until they converge at the user-defined criterion. This algorithm is attractive because of its simplicity and accuracy [15]. However, the original ICP algorithm assumes outlier-free data and $100 \%$ overlap of the model and data points [16]. These assumptions are not applicable for construction applications because the point cloud obtained from the construction site contains noise and only partially corresponds with the 3D CAD model.

An algorithm that can improve these limitations is the Levenberg-Marquardt iterative closest point (LM-ICP) algorithm [15]. To generate the correct correspondence of noisy data, LM-ICP serves as the threshold and weights are introduced to deal with the issue of data points having no correspondence with model points.

Below is the LM-ICP algorithm. Let $a$ be the transformation parameter (rotation matrix $R$ and translation vector $t$ ); then, the transformation can be defined as:

$$
T(a ; x)=R x+t
$$

The objective of the registration is to obtain transformation parameters that minimize the distance between the data and model points. Thus, the error function to be minimized is defined as:

$$
E(a)=\sum_{i=1}^{N} \epsilon^{2}\left(\left|m_{i}-T\left(a ; d_{i}\right)\right|\right)
$$

where $m_{i}$ is the model points and $d_{i}$ refers to the data points.

To reduce false correspondence caused by noise, the user can define the threshold value which serves to make the correspondence robust to noise. In addition, when dealing with data points for which there is no correspondence with the model points, weights $w_{i}$ are set to zero for points with no match; otherwise, one is introduced. Thus, the error function to be minimized is:

$$
E(a)=\sum_{i=1}^{N} w_{i} \epsilon^{2}\left(\left|m_{i}-T\left(a ; d_{i}\right)\right|\right)
$$

To solve the least squares problem, the LevenbergMarquardt algorithm uses a gradient descent to minimize the error function. It can be represented as follows:

$$
\nabla_{\delta} E(a+\delta)=\mathrm{J}^{\mathrm{T}} \mathrm{e}+\mathrm{J}^{\mathrm{T}} \mathrm{J} \delta=0
$$

where $a$ is the transformation parameter, $a+\delta$ is the new estimated transformation parameter, and J is the Jacobian matrix with $\mathrm{J}=\frac{\partial E}{\partial a}$.

The Levenberg-Marquardt algorithm updates $\delta$ to reduce error and can be used to determine the transformation parameter through repetitive updates:

$$
\delta=-\left(\mathrm{J}^{\mathrm{T}}+\lambda \mathrm{I}\right)^{-1} \mathrm{~J}^{\mathrm{T}} \mathrm{e}
$$


where $\lambda$ is set to a start value and is updated in each iteration and, I is an identity matrix.

\section{EXPERIMENTAL RESULTS}

Field experiments were conducted at the construction site in order to establish the validity of the proposed automated registration method. In the field experiments, a concrete building under construction was chosen to test the proposed method's applicability to progress monitoring and as-built modeling. From 2D CAD drawings, a 3D CAD model for the entire structure was generated, as illustrated in Fig. 1 (a). In order to obtain a complete point cloud of the structure, the commercial photogrammetric system Photomodeler Scanner, developed by Eos Systems, Inc., was adopted [17] and 921,782 points were obtained from 12 images. Point cloud from the construction site using photorgrammetry is illustrated in Fig. 1 (b).

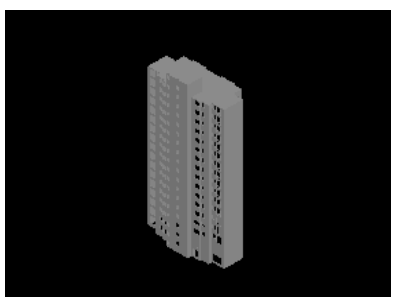

(a)

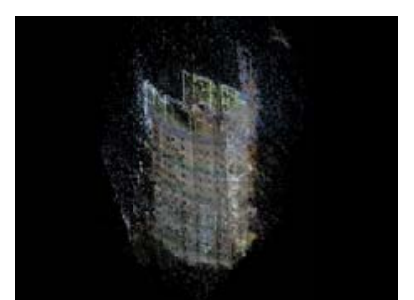

(b)
Fig. 1 The test data sets used for experiments (a) 3D CAD model (b) point cloud from the construction site

To perform registration, the 3D CAD model was converted to STL format, then 125,957 model points were generated. In data re-sampling, both model and data points were refined at the same resolution using octree. The model and data composed 22,522 points and 19,015 points, respectively. Fig. $\quad 2$ (a) and (b) show full and top views of the resampled model points, and (c) and (d) show full and top views of the resampled data points.
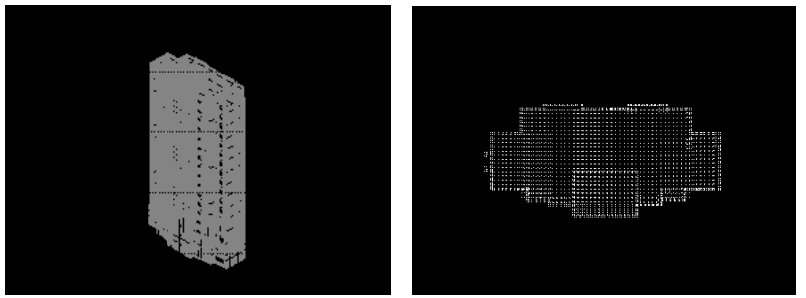

(a)

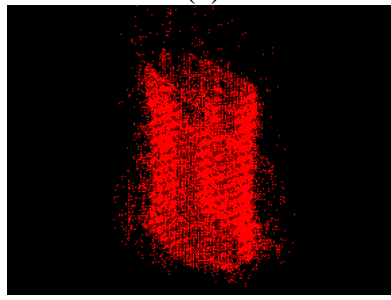

(c) (b)

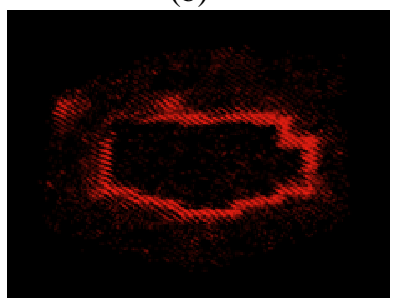

(d)
Fig. 2 Results of data resampling (a) Full view of the resampled model points (b) Top view of the resampled model points (c) Full view of the resampled data points (d) Top view of the resampled data points

To align the coordination system of model and data points that belong to each position, the registration process, which consists of coarse and fine registration, was performed. The rotation angle $\left(-127.7448^{\circ},-47.4038^{\circ}, 30.4078^{\circ}\right)$ and translation vector (81195.7187, -123636.6556, -8152.2892) were obtained from the PCA based coarse registration. As Fig. 3 (a) and (b) demonstrate, PCA based coarse registration provides rough registration results which can create difficulties when performing precision progress monitoring or as-built modeling. Thus, fine registration is required to improve coarse registration results.

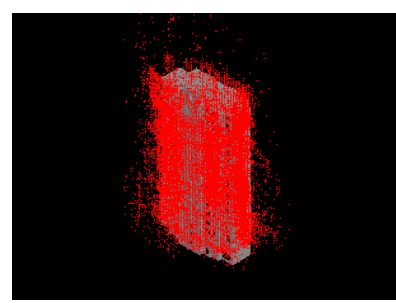

(a)

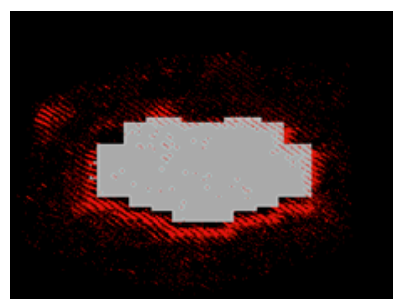

(b)
Fig. 3 Result of PCA based coarse registration (a) Full view (b) Top view

Fig. 4 (a) and (b) show the result of LM-ICP based fine registration from which the rotation angle $\left(-1.8313^{\circ}\right.$, $\left.0.6240^{\circ},-2.8547^{\circ}\right)$ and translation vector $(-530.1868$, 609.9831, -2906.6546) were obtained. The registration procedure is fully automated, and the result shows that the proposed method is robust to noisy point cloud obtained from the construction site. 


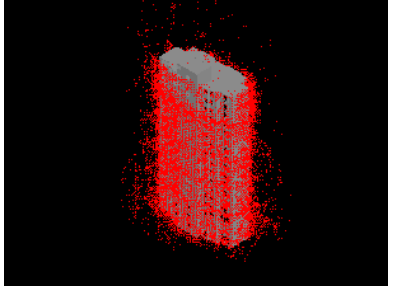

(a)

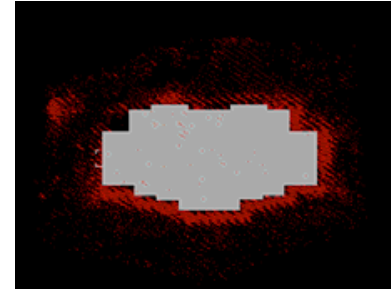

(b)
Fig. 4 Result of LM-ICP based fine registration (a) Full view (b) Top view

\section{CONCLUSION}

This paper presented a fully automated 3D registration method for use in determining the transformation parameters of a 3D CAD model with a point cloud obtained from the construction site. The proposed method has the advantages of full automation and robustness. The experimental results show that the proposed method can successfully determine the transformation parameters to align the 3D CAD model and noisy point cloud. The proposed method facilitates performing registration of 3D CAD model with point cloud obtained from the construction site without manual intervention. Thus, it is possible to obtain accurate registration results efficiently, and potentially contributes to the construction automation applications in terms of registration. Future research will include tests of the proposed methods in practical applications, including progress monitoring or as-built modeling.

\section{ACKNOWLEDGEMENTS}

This research was supported by Basic Science Research Program through the National Research Foundation of Korea (NRF) funded by the Ministry of Education, Science and Technology (2010-0023229).

\section{REFERENCES}

[1] Lu, Y., Liao, Z.W., and Chen, W.F., “An Automatic Registration Framework Using Quantum Particle Swarm Optimization for Remote Sensing Images”, Proc., of the International Conference on Wavelet Analysis and Pattern Recognition, Beijing, China, 2007.
[2] Zhao, J., and Goonetilleke, R.S., "Locating Anatomical Points on Foot from 3D Point Cloud Data”, Proc., of the $16^{\text {th }}$ International Conference on Artificial Reality and Telexistence, Hangzhou, China, 2006.

[3] Meshoul, S., and Batouche, M., “A Fully Automatic Method for Feature-Based Image Registration”, Proc., of the IEEE International Conference on Systems, Man and Cybernetics, Hammamet, Tunisia, 2002.

[4] Oertel, C., Colder, B., Colombe, J., High, J., Ingram, M., and Sallee, P., "Current Challenges in Automating Visual Perception", Proc., of the 37th IEEE Applied Imagery Pattern Recognition Workshop, Washington, DC, 2008.

[5] Shih, N.J., and Wang, P.H., "Point-Cloud-Based Comparison between Construction Schedule and As-Built Progress: Long-Range Three-Dimensional Laser Scanner's Approach”, Journal of Architectural Engineering, Vol. 10(3), pp. 98-102, 2004.

[6] Bosche, F., “Automated Recognition of 3D CAD Model Objects in Laser Scans and Calculation of As-Built Dimensions for Dimensional Compliance Control in Construction”, Advanced Engineering Informatics, Vol. 24(1), pp. 107-118, 2010.

[7] Son, H., and Kim, C., “3D Structural Component Recognition and Modeling Method Using Color and 3D Data for Construction Progress Monitoring”, Automation in Construction, Vol. 19(7), pp. 844-854, 2010.

[8] Dai, X., and Khorram, S., “A Feature-Based Image Registration Algorithm Using Improved Chain-Code Representation Combined with Invariant Moments”, IEEE Transactions on Geoscience and Remote Sensing, Vol. 37(5), pp. 2351-2362, 1999.

[9] Li, W., Yin, Z., Huang, Y., and Xiong, Y., “Automatic Registration for 3D Shapes Using Hybrid DimensionalityReduction Shape Description”, Pattern Recognition, Article in Press, 2011. 
[10] Lee, K.H., Woo, H., and Suk, T., "Point Data Reduction Using 3D Grids”, The International Journal of Advanced Manufacturing Technology, Vol. 18(3), 201-210, 2001.

[11] Chung, D.H., Yun, Y.D., and Lee, S.U., “Registration of Multiple-Range Views Using the Reverse-Calibration Technique”, Pattern Recognition, Vol. 31(4), pp. 457-464, 1998.

[12] Salvi, J., Matabosch, C., Fofi, D., and Forest, J., “A Review of Recent Range Image Registration Methods with Accuracy Evaluation”, Image and Vision Computing, Vol. 25(5), pp. 578-596, 2007.

[13] Besl, P.J., and McKay, N.D., “A Method for Registration of 3-D Shapes”, IEEE Transactions on Pattern Analysis and Machine Intelligence, Vol. 14(2), pp. 239-256, 1992.

[14] Du, S., Zheng, N., Ying, S., and Liu, J., “Affine Iterative Closest Point Algorithm for Point Set Registration”, Pattern Recognition Letters, Vol. 31(9), pp. 791-799, 2010.

[15] Fitzgibbon, A.W., "Robust Registration of 2D and 3D Point Sets”, Image and Vision Computing, Vol. 21(13-14), pp. 1145-1153, 2003.

[16] Lomonsov, E., Chetverikov, D., and Ekart, A., "PreRegistration of Arbitrarily Oriented 3D Surfaces Using a Genetic Algorithm”, Pattern Recognition Letters, Vol. 27(11), 1201-1208, 2006.

[17] Eos Systems, Inc. http://www.photomodeler.com/index.htm, last accessed on April 122010. 